Itinéraires Itinéraires

Littérature, textes, cultures

2013-1 | 2013

La fiction aujourd'hui

La fiction à l'épreuve du jeu de rôle. Oliver Caïra, Jeux de rôle, les forges de la fiction

Paris, CNRS Éditions, 2007, 311 pages

\title{
Geoffroy Brunson
}

\section{OpenEdition}

Journals

Édition électronique

URL : http://journals.openedition.org/itineraires/891

DOI : 10.4000/itineraires.891

ISSN : 2427-920X

Éditeur

Pléiade

Édition imprimée

Date de publication : 1 octobre 2013

Pagination : 197-200

ISBN : 978-2-343-01791-4

ISSN : 2100-1340

\section{Référence électronique}

Geoffroy Brunson, "La fiction à l'épreuve du jeu de rôle. Oliver Caïra, Jeux de rôle, les forges de la fiction », Itinéraires [En ligne], 2013-1 | 2013, mis en ligne le 01 octobre 2013, consulté le 22 septembre 2020 URL : http://journals.openedition.org/itineraires/891 ; DOI : https://doi.org/10.4000/itineraires.891

Ce document a été généré automatiquement le 22 septembre 2020.

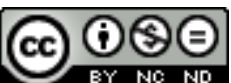

Itinéraires est mis à disposition selon les termes de la licence Creative Commons Attribution - Pas d'Utilisation Commerciale - Pas de Modification 4.0 International. 


\section{La fiction à l'épreuve du jeu de rôle. Oliver Caïra, Jeux de rôle, les forges de la fiction}

Paris, CNRS Éditions, 2007, 311 pages

\section{Geoffroy Brunson}

\section{RÉFÉRENCE}

Oliver Caïra, Jeux de rôle, les forges de la fiction, Paris, CNRS Éditions, 2007, 311 pages

Méconnu du grand public, encore marqué par les errements médiatiques des années 1990, le jeu de rôle n'a que rarement intéressé les chercheurs en sciences humaines, qui l'ont alors utilisé comme «analyseur du social» (p. 7). Olivier Caïra, sociologue et rôliste confirmé, prend ses distances avec cette démarche et propose d'examiner le jeu de rôle comme un objet sociologique à part entière dans un ouvrage qui, centré sur l'expérience fictionnelle propre à ce medium, unit avec réussite outils et méthodes issus des sciences sociales et théories de la fiction.

2 Les forges de la fiction se présente comme un ouvrage modulaire - « un livre dont vous êtes le héros »- suggérant aux lecteurs divers parcours de lecture selon le profil (néophyte ou rôliste) et les objectifs (découverte ou approfondissement universitaire) de chacun. Les dix chapitres sont répartis en trois grandes parties : la première dresse un vaste historique du jeu de rôle et en présente les composantes essentielles; la deuxième propose une approche ethnographique du jeu de rôle, l'auteur décrivant et analysant trois séances préparées par ses soins et jouées en compagnie de groupes diversement familiarisés au jeu de rôle ; la troisième consiste en l'étude serrée du jeu de rôle comme formule d'engagement fictionnel spécifique où les participants « interrogent tous les présupposés de la description, tous les mécanismes de l'action au sein de l'univers » fictionnel (p. 8). 
3 La première partie, "Un nouveau type de jeux de société », détaille les origines et l'histoire du jeu de rôle, en décrit les genres et univers principaux, puis met en lumière les spécificités de l'activité éditoriale d'un secteur caractérisé par une série de contraintes économiques et par un rapport singulier à son public qui le situent au cœur d'une proto-industrie des loisirs en marge du secteur du divertissement. Olivier Caïra expose ensuite les différentes composantes d'une partie, et souligne la tâche complexe du maitre de jeu, à qui il appartient de réaliser un «montage » unissant de manière cohérente l'univers de jeu, le système de règles, le scénario, les personnages - montage qui devra satisfaire les attentes et compétences des différents joueurs, quitte à modifier ou ignorer le système de règles. Le jeu de rôle apparaît ainsi comme une « construction fictionnelle» (p. 70), une expérience dont ne peut rendre compte la seule description des éléments du montage, ce qui mène le chercheur à envisager une approche ethnographique.

Dans la deuxième partie, "Ethnographie d'une séance ", Olivier Caïra présente et analyse, en s'appuyant sur de nombreux extraits, la préparation et le déroulement d'une séance de jeu de rôle. Examinant les trois phases de jeu - préparation, aventure, clôture - auprès de chacun des trois groupes, l'auteur met d'abord en évidence les "dénouements très différents malgré une base scénaristique » identique (p.116), qui illustrent l'importante plasticité narrative du jeu de rôle; il montre ensuite qu'au-delà de la dyade victoire/défaite, les critères d'évaluation et de réussite d'une partie sont nombreux, propres à chaque groupe, et échappent à la calculabilité des jeux traditionnels. En tant qu'expérience fictionnelle, le jeu de rôle se fonde en réalité « sur une esthétique complexe » et repose sur une série de " conventions de transcription »le concept est emprunté à Goffman - qui permettent la "transformation de l'activité supposée des personnages en une séquence de jeu de rôle». Olivier Caïra se penche donc, pour terminer, sur les conventions relatives à la gestion du temps, à l'action et au récit, ce qui le mène à définir le jeu de rôle comme une création fictionnelle improvisée, collective, qui cherche «sa cohérence dans un ensemble de références et de niveaux de discours hétérogènes" (p.141). Dans ce contexte, le meneur de jeu possède un rôle essentiel : réguler une série de « carrefours » de la fiction.

5 La troisième partie, «Le jeu de rôle à la croisée des fictions », est centrée sur l'analyse pointue de ces carrefours; le sociologue en dénombre cinq.

1. Carrefour des univers: les mondes du jeu de rôle constituent une composition: gouvernés par une pulsion de complétude inassouvie, ils comblent les lacunes de la description verbale par des ressources documentaires ou fictionnelles. Il appartient au meneur de jeu (MJ) d'assimiler les références venues de tous horizons, et de faire cohabiter les représentations des différents joueurs.

2. Carrefour des fonctions créatives: l'engendrement de l'univers de jeu est une co-création qui révèle une division des tâches, le $\mathrm{MJ}$ revêtant une fonction d'animateur chargé d'arbitrer les litiges créatifs et de résoudre les débats. Le jeu de rôle, à cet égard, rend poreuses les "barrières classiques entre "producteurs" et "récepteurs" de l'expression fictionnelle " (p. 167).

3. Carrefour des niveaux de communication : les séances de jeu de rôle sont marquées par un va-et-vient constant entre engagement et distanciation par rapport à la fiction, entre premier et second degré, entre monde actuel et monde fictionnel. Les joueurs, toujours dans le monde fictif et autour de la table, jonglent entre les niveaux de communication et les degrés de fictionnalité. Il appartient dès lors au MJ de distinguer entre sérieux et dérision. 
4. Carrefour des règles d'engendrement : le jeu de rôle repose sur la tension entre l'infinité des situations à simuler et la finitude des règles de simulation, et entre ces dernières et les représentations que les joueurs se font du monde fictionnel. Si elles modèlent l'expérience fictionnelle - en proposant des lois physiques et scientifiques, en définissant une anthropologie, en prescrivant sanctions et récompenses -, les règles apparaissent avant tout comme une boîte à outils qui permet aux joueurs, et en particulier au MJ, d'improviser à partir d'instruments spécialisés mais flexibles, généraux mais adaptables aux situations ponctuelles.

5. Carrefour des exigences ludiques : les règles du jeu de rôle, contrairement au jeu de société, ne prescrivent pas de conditions de victoire. La réussite d'une partie repose sur un contrat, un consensus entre joueurs, chacun possédant ses exigences et ses principes d'évaluation. Proposant un vaste panorama des diverses exigences qui structurent l'expérience fictionnelle, le chercheur montre qu'il appartient au MJ de concilier au mieux les attentes et exigences de chacun.

6 Concluant, Olivier Caïra décrit le jeu de rôle comme une composition collective et réflexive, située entre description et exécution, et « dans laquelle chaque séquence est soumise en temps réel à l'appréciation de l'équipe ». C'est cette complexité, l'emboîtement constant des "modes de communication hétérogènes » (p. 259) qui, selon le chercheur, pourrait expliquer les difficultés qu'éprouve le grand public à comprendre l'expérience ludique du jeu de rôle ; celui-ci serait caractérisé avant tout par la mise en œuvre complexe de plusieurs compétences permettant de construire une fiction partagée par le seul biais de la parole, et définissant la «formule d'engagement " des joueurs, leur décision d'entrer dans un univers fictionnel par le biais du jeu de rôle. L'ouvrage se termine par un appendice dans lequel l'auteur de Hollywood face à la censure souligne les faiblesses des deux autres principales publications francophones consacrées au jeu de rôle ${ }^{1}$, puis expose et justifie ses choix méthodologiques, en particulier l'articulation des postures de rôliste et de sociologue du jeu de rôle.

7 Olivier Caïra s'était fixé un double objectif au seuil des Forges de la fiction : proposer une étude minutieuse du jeu de rôle en tant qu'expérience fictionnelle, ainsi qu'un ouvrage accessible à un large lectorat. Trois cents pages plus loin, il est indéniable que le chercheur a réussi dans son entreprise. Dans les dernières lignes, celui-ci souhaite que son travail «suscit[e] des vocations de recherche " sur le jeu de rôle, mais affirme ne pas chercher à "s'imposer comme référence » (p. 269) dans le domaine. Si sociologues et théoriciens de la fiction gagneront à exaucer le premier vœu de Caïra, nous décevrons le second: cet ouvrage est à l'heure actuelle une référence francophone incontournable sur le jeu de rôle.

\section{NOTES}

1. Didier Guiserix, Le Livre des jeux de rôle, Paris, Bornemann, 1997 ; Laurent Trémel, Jeux de rôles, jeux vidéo, multimédia, les faiseurs de mondes, Paris, PUF, 2001. 


\section{AUTEURS}

GEOFFROY BRUNSON

Asp. F.R.S.-FNRS, Université catholique de Louvain 